Mots. Les langages du politique

112 | 2016

Discours présidentiels et de présidentielles

\title{
Le genre des présidentielles. Femmes et hommes en campagne
}

Gender and presidential elections. Women and men in campaign

Género y elecciones presidenciales. Mujeres y hombres en campaña

Marlène Coulomb-Gully

(2) OpenEdition

Journals

Édition électronique

URL : https://journals.openedition.org/mots/22429

DOI : $10.4000 /$ mots. 22429

ISSN : 1960-6001

Éditeur

ENS Éditions

Édition imprimée

Date de publication : 15 octobre 2016

Pagination : 29-36

ISBN : 978-2-84788-850-8

ISSN : 0243-6450

Référence électronique

Marlène Coulomb-Gully, « Le genre des présidentielles. Femmes et hommes en campagne », Mots. Les langages du politique [En ligne], 112 | 2016, mis en ligne le 15 octobre 2018, consulté le 22 avril 2022 URL : http://journals.openedition.org/mots/22429 ; DOI : https://doi.org/10.4000/mots.22429

(C) ENS Éditions 


\title{
Le genre des présidentielles. Femmes et hommes en campagne
}

\author{
Je peux bien vous faire vibrer avec « la grandeur de la France, phare \\ millénaire... » Je peux très bien le faire, vous faire de beaux discours, avec des \\ morceaux de bravoure. Mais je crois que les gens n'en ont plus rien à faire. \\ L'image du Père de la nation guidant son peuple vers \\ la lumière, c'est un peu usé, non ? Il va falloir passer d'Hugo à Prévert. \\ La présidente, dans L'État de grâce, 2006
}

Passer d'Hugo à Prévert : telle serait la marque du féminin dans le discours politique... tout au moins à en croire les scénaristes de cette série télévisée ${ }^{1}$ Il n'est pas anodin que notre entrée en matière s'effectue par le biais d'une fiction, aucune femme n'ayant en France accédé à la magistrature suprême. Car si le genre ne saurait se limiter à l'étude des femmes, celles-ci ont cependant permis la prise de conscience du caractère sexué de l'exercice du pouvoir - que nous entendrons ici au sens restreint d'exercice institutionnel des fonctions politiques.

Si, et on ne saurait s'en étonner, les politistes ont été pionniers dans ces travaux, les historiens et historiennes, les sociologues, les linguistes, les spécialistes de communication, etc., s'en sont progressivement emparés, construisant un objet de recherche fortement marqué par l'interdisciplinarité.

Soulignons également la force d'imposition du calendrier politique dans l'agenda de ces travaux, avec les débats autour de la parité dans les années quatre-vingt-dix puis la candidature de Ségolène Royal en 2007, avant que ceux-ci ne s'inscrivent de façon durable dans l'analyse du champ politique².

\section{L’imaginaire du pouvoir ou l'exclusion des femmes}

La Cinquième République fait de l'élection à la présidence de la République le point de convergence des stratégies des partis et des candidats et candidates

1. Diffusée sur France 2 en 2006, durant les primaires socialistes qui ont vu la victoire de Ségolène Royal, L'État de grâce met en scène une femme présidente de la République.

2. Nous nous limiterons, dans le cadre de cet article, aux travaux produits dans l'espace français. 
comme de la mobilisation citoyenne. Jusqu'en 2007, les femmes ont été cantonnées au rôle de figurantes - candidates de témoignage, militantes, épouses des candidats -, une "anomâlie» qu'explorent les travaux sur la «démocratie exclusive» instaurée par la Révolution (Fraisse, 1989) et la tension dans les définitions de la citoyenneté et du féminin (Scott, 1998). L'imaginaire politique s'est ainsi construit contre les femmes et le féminin (Derville, Pionchon, 2004; Le Bras-Choppard, 2004; Viennot, 2006 et 2008), les excluant de fait de la compétition présidentielle d'abord perçue comme un combat de chefs. Le déni de représentativité est au cœur de leur exclusion des fonctions électives (face au masculin construit comme neutre et universel, le féminin apparaît comme spécifique), alors qu'elles sont de plus en plus nombreuses au sein de l'exécutif des gouvernements, un paradoxe qui met en lumière l'opposition compétence/représentation centrale dans notre problématique (Sineau, 1988 et 2011).

\section{Femmes en campagne}

Dans les années quatre-vingt-dix, les débats autour du thème de la parité en politique donnent lieu à des travaux qui, portés par une nouvelle génération de chercheurs et de chercheuses, renouvellent les questions posées en mettant l'accent sur les usages et les effets contradictoires de l'argument de genre: considérées comme un atout dans les campagnes de proximité (les municipales), les qualités réputées féminines comme le sens du concret, le souci de l'autre, une moindre ambition, etc., écartent les femmes des positions de pouvoir (Fassin, Guionnet éd., 2002).

Ces travaux constituent autant de jalons dans la problématisation du genre lors de la présidentielle de 2007.

Cette élection qui voit pour la première fois une femme candidate d'un parti de gouvernement permet d'abord un retour sur l'ensemble des femmes qui se sont portées candidates sous la Cinquième République : des candidates de témoignage, présentées par des petits partis qui voient là l'occasion de se démarquer stratégiquement (Coulomb-Gully, 2012; Sineau, 2008). Ainsi d'Arlette Laguiller - recordwoman des présidentiables - pour Lutte ouvrière, ou de Christiane Taubira - femme et noire - pour le Parti radical de gauche. Parmi les thématiques que développent ces recherches, signalons l'attention portée au corps des candidats et candidates, comme si la présence d'une femme révélait - au sens chimique du terme - la centralité de cet opérateur politique : proximité/ distance, horizontalité/hiérarchie, «care »/autorité, usages de la maternité et de la beauté sont réévalués à cette occasion (Achin, Dorlin, Rennes éd., 2008) tandis qu'est revisitée la notion d'ethos articulant le physique, la voix, le vêtement, la gestualité et les propos des candidats et candidates (Coulomb-Gully, 2009). 


\section{La fabrique médiatique}

Les médias sont un objet d'investigation classique en politique en raison de leur rôle d'animation de l'espace public, tout particulièrement lors de ces moments de surchauffe électorale que sont les présidentielles. Les travaux sur le genre soulignent leur caractère structurellement sexiste (Bertini, 2002), dont témoignent en particulier les dissymétries d'images et de formulations véhiculées à cette occasion, et qui contribuent le plus souvent à disqualifier les femmes de la compétition électorale. La compétence des candidates, au cœur de la construction de leur légitimité politique, se trouve ainsi régulièrement mise en cause : l'accent mis sur leur apparence physique, leur vie privée, leurs engagements familiaux, leur aptitude au "care», etc. dessine en creux les caractéristiques plus régaliennes nécessaires à l'exercice du pouvoir (Coulomb-Gully éd., 2009; Olivesi, 2012). Sans que le concept de genre soit explicitement mobilisé, mentionnons néanmoins l'étude de la «présidentiabilité» de François Hollande et Martine Aubry lors des primaires socialistes de 2011 (Baider, 2015).

Le travail des journalistes et leurs contraintes professionnelles ont également été revisités dans une perspective genrée (Damian-Gaillard, Frisque, Saïtta éd., 2010) : après la campagne de 2007 où les médias ont tour à tour été accusés d'avoir créé de toutes pièces la "bulle Royal» puis d'avoir contribué à l'échec de la candidate, des travaux portant sur la présidentielle de 2012 mettent l'accent sur la routinisation des procédures qui favorise leur caractère gender-blind et aboutit à la reconduction des stéréotypes, et ce alors même que les rédactions sont désormais largement féminisées (Chauvel, Le Renard, 2013).

\section{Masculinités et hétérosexualité normative}

La mise en évidence du caractère construit de la féminité a permis de s'interroger en retour sur ce qu'est être un homme politique. En effet, impensée jusqu'à l'arrivée des femmes en politique, la présidentialité des candidats s'est construite sur un implicite masculin caractéristique de l'ethos bourgeois des $19^{\mathrm{e}}$ et $20^{\mathrm{e}}$ siècles, fondé sur des valeurs comme la droiture, le sens de la responsabilité et du collectif, le courage, l'autorité, etc., l'entrée dans l'ère paritaire transformant cette "virilité privilège » en «virilité ressource» (Achin et al., 2007).

Le face-à-face Royal-Sarkozy lors de la présidentielle de 2007 a fait émerger la problématique, la féminité de la candidate soulignant le caractère construit de sa virilité par le candidat. La campagne de 2012, qui marque le retour à la "normâle» avec le vis-à-vis de deux hommes au second tour, permet le développement et l'approfondissement de ce thème. Sont analysés sous cet angle 
les jeux de concurrence entre François Hollande et Nicolas Sarkozy, pour imposer une définition de la virilité en phase avec la notion de présidentialité dans le contexte d'alors (Coulomb-Gully, 2013), et la masculinité de François Bayrou, prisonnier des injonctions contradictoires peut-être liées à une «impossible virilité centriste » (Arambourou, 2013).

L'hétérosexualité normative qui sous-tend ces modèles de masculinité permet un retour sur le rôle de "femme de », l'instrumentalisation de cet «accessoire marital» ayant été perçu dès les premières présidentielles (RestierMelleray, 1999; Le Bras-Choppard, 2009).

\section{Race, classe, genre : jeux de rôles}

La campagne présidentielle étasunienne de 2008 constitue un cas d'école pour les travaux sur le genre : en effet, lors des primaires démocrates, l'éviction d'Hillary Clinton, femme et blanche, s'est faite au profit de Barack Obama, homme et noir. L'articulation race/classe/genre (à laquelle il faudrait ajouter l'âge) est au cœur de l'approche en termes d' «intersectionnalité » qui permet d'éclairer le vis-à-vis des deux candidats (Fassin, 2008).

La France, excepté la candidature déjà mentionnée de Christiane Taubira pour le Parti radical de gauche en 2002, n'a compté aucun candidat ou candidate noir à la présidentielle. La présence de «personnes de couleur» dans les spots de campagne (Borell, 2014) et plus encore le rôle des porte-paroles «issues de la diversité » ont été analysés comme des identités de complément : qu'il s'agisse, pour cette dernière fonction, de femmes face à un homme pour contrer l'entre-soi masculin et faire preuve de bonne volonté paritaire (Bard, 2012) ou de femmes issues de la diversité face à des présidentiables blancs. En 2007, Rachida Dati pour Nicolas Sarkozy et Najat Vallaud-Belkacem pour Ségolène Royal, ou le couple contrasté formé en 2012 par Nathalie KosciuskoMorizet pour Nicolas Sarkozy et Najat Vallaud-Belkacem pour François Hollande (Paoletti, 2013), illustrent une fois encore l'intérêt de la démarche intersectionnelle.

\section{Le discours présidentiel dans l’angle mort}

Si, dans leurs discours, certains candidats et candidates s'emploient à relayer les revendications liées au genre et à la sexualité (accès à la contraception, divorce par consentement mutuel, légalisation de l'avortement, dépénalisation de l'homosexualité, PACS, parité, «mariage pour tous», etc. - Sineau, 2008), la dimension genrée des discours proprement dits a été peu travaillée.

Les normes du discours politique sont implicitement viriles, une dimension qui exclut «naturellement» les femmes de l'exercice oratoire et contribue sans 
doute à les disqualifier dans la conquête du pouvoir. Pourtant, alors que les discours de présidentielles (discours de présidents, déclarations de candidatures, campagnes officielles, débats d'entre-deux-tours, discours d'investiture, etc.) ont suscité d'innombrables travaux, peu d'entre eux portent sur la dimension genrée de l'exercice. Hors du cadre présidentiel stricto sensu, signalons toutefois les travaux du CEDITEC sur les discours des ministres du gouvernement Jospin (1997-1999 en particulier) : en croisant diverses approches méthodologiques, ils concluent, quoique avec prudence, à une convergence entre ce qu'ils nomment «le modèle pragmatique-empathique » et un possible ethos féminin (Bonnafous, 2003; Vassy, 2005). Mentionnons également la colossale recherche sur les professions de foi des députées sous la Cinquième République (Guaresi, 2015) qui, outre le moindre recours au vocabulaire politique institutionnel et la prégnance de la thématique socio-économique, révèle surtout leur caractère faiblement sexué (sur un corpus plus restreint, voir également Lévêque, 2005).

Le caractère masculino-centré de la langue constitue en revanche un champ de recherche largement investi, qui rappelle que la domination politique est aussi linguistique (Moreau, Baider, Kaznadar éd., 2007). Jusqu'en 2007, la campagne officielle à l'élection présidentielle ne s'intitulait-elle pas «Campagne officielle pour l'élection du président de la République»?

\section{Le « genre » de la fiction}

Le discours des fictions est partie prenante de la réalité, comme l'imaginaire appartient au réel. La légitimation des séries télé comme œuvres à part entière depuis la fin du $20^{e}$ siècle en a fait des objets d'investigation pour les chercheurs et chercheuses, en même temps que le thème du président devenait un motif privilégié. Si la question du genre n'y est pas explicitée lorsqu'y sont mis en scène des hommes politiques, elle est au cœur du propos lorsqu'il s'agit d'une femme, ces fictions fonctionnant comme le lieu d'expression par excellence des dynamiques et des tensions à l'œuvre dans la société dont elles sont l'expression. Ainsi des séries américaines Commander in Chief ou $24 \mathrm{~h}$ chrono diffusées lorsqu'était attendue la candidature d'Hillary Clinton, de la série danoise Borgen ou des séries françaises L'État de grâce ou Les hommes de l'ombre, lorsque Ségolène Royal puis Martine Aubry apparaissaient comme de possibles présidentiables (Coulomb-Gully, 2014). Le réalisme revendiqué de ces séries s'est trouvé démenti par la réalité des faits...

La pensée du genre renouvelle en profondeur l'approche $d u$ politique comme de la politique. Les recherches réalisées depuis les années quatre-vingt ont révélé l'impensé que constituait la masculinité, au cœur de la construction de la légitimité politique en général et présidentielle en particulier, en même temps qu'elles ont exploré les ambivalences des usages de la féminité. 
La présidentielle de 2012 confirme l'enracinement des travaux sur le genre, qu'on aurait pu croire limités à l'exception constituée par la candidature de Ségolène Royal en 2007 (Barros, 2013). Soulignons pour finir la réflexivité par rapport au genre, dont les politiques font désormais preuve dans leurs stratégies de campagne, et qui témoigne du fort niveau de «percolation » des travaux sur le genre dans la société actuelle. Comme l'affirment Catherine Achin et Lucie Bargel (2013), le genre deviendrait ainsi un des premiers langages du politique...

\section{Références}

ACHIN Catherine, BARgel Lucie, 2013, "Montrez ce genre que je ne saurais voir". Genre, sexualité et institutions dans la présidentielle de 2012 », Genre, sexualité \& société, Hors-série n ${ }^{\circ} 2$, «Présidentielle 2012 ».

Achin Catherine, Dorlin Elsa, Rennes Juliette éd., 2008, Raisons politiques nº31, mars, "Le corps présidentiable».

ACHIN Catherine et al., 2007, Sexes, genre et politique, Paris, Economica.

Arambourou Clément, 2013, «De la masculinité de François Bayrou. Une analyse en creux des conditions d'efficacité d'un registre identitaire controversé», Genre, sexualité \& société, Hors-série n ${ }^{2} 2$, «Présidentielle 2012 ».

BAIDER Fabienne, 2015, "Évaluation de la présidentiabilité (à l'interface de la sémantique lexicale et de la linguistique de corpus)», Mots. Les langages du politique, $n^{\circ} 108$, «Thèmes et thématiques dans le discours politique », p. 103-128.

BARD Christine, 2012, "Performances de genre. Images croisées de Michèle AlliotMarie et de Roselyne Bachelot », Histoire@Politique, nº 17, p.69-86.

BARRoS Françoise (de), 2013, "Les jeux sur le genre : retour à la normâlité», Genre, sexualité \& société, Hors-série n ${ }^{2} 2$, "Présidentielle 2012».

BERTINI Marie-Joseph, 2002, Femmes, Le pouvoir impossible, Paris, Pauvert.

BonnAfous Simone, 2003, " “Femmes politiques". Une question de genre?», Réseaux, $\mathrm{n}^{0} 120$, p. 121-145.

Borell Alexandre, 2014, «Un “corps français” traditionnel? Usage de la couleur de la peau dans les spots des candidats à la présidentielle (1988-2007)», Sous les images, la politique..., I. Veyrat-Masson, S. Denis, C. Sécail éd., Paris, CNRS, p. 195210.

Chauvel Séverine, Le RenARd Amélie, 2013, «Comment le travail journalistique amplifie la hiérarchie de genre. Une rédaction pendant la campagne présidentielle de 2012 », Genre, sexualité \& société, Hors-série n 0 2, « Présidentielle 2012 ».

Coulomb-Gully Marlène, 2009, "Le corps présidentiel», Mots. Les langages du politique, n 89, 2007, «Débats pour l’Élysée », p. 25-38.

- 2012, Présidente, le grand défi. Femmes, politique et médias, Paris, Payot.

— 2013, "Mâle ou normal?". Incarnation et masculinité(s) du couple Hollande-Sarkozy dans la campagne présidentielle de 2012 », Genre, sexualité \& société, Horssérie $n^{\circ} 2$, «Présidentielle 2012 ». 
- 2014, "Femmes politiques : une fiction? », I. Veyrat-Masson, S. Denis, C. Sécail éd., Sous les images, la politique..., Paris, CNRS, p.17-28.

Coulomb-Gully Marlène éd., 2009, «Présidentielle 2007. Scènes de genre », Mots. Les langages du politique, $\mathrm{n}^{\circ}$ 89, « 2007. Débats pour l’Élysée », p. 25-38.

Damian-Gaillard Béatrice, Frisque Cégolène, SAÏTTA Eugénie éd., 2010, Le journalisme au féminin. Assignations, inventions et stratégies, Rennes, PUR.

DervilLe Grégory, PIonchon Sylvie, 2004, Les femmes et la politique, Grenoble, PUG.

FASSIN Éric, 2008, «Des identités politiques. Jeux et enjeux du genre et de la race dans les primaires démocrates aux États-Unis en 2008 ", Raisons politiques, mars, $\mathrm{n}^{\circ}$ 31, p. 65-79.

FASSIn Éric, GuiOnNet Christine éd., 2002, Politix, vol. 15, n 60, La parité en pratiques.

FraISSE Geneviève, 1989, Muse de la raison. Démocratie et exclusion des femmes en France, Paris, Gallimard.

GUARESI Magali, «Les thèmes dans le discours électoral de candidature à la députation sous la Cinquième République. Perspective de genre (1958-2007)», Mots. Les langages du politique, $\mathrm{n}^{\circ} 108$, "Thèmes et thématiques dans le discours politique ", p. 15-37.

Le Bras-Choppard Armelle, 2004, Le masculin, le sexuel, le politique, Paris, Plon.

- 2009, Première dame, second rôle, Paris, Le Seuil.

LÉvÈQUE Sandrine, 2005, "La féminité “dépassée”? Usages et non-usages du genre dans les professions de foi des candidat(e)s parisien(ne)s aux élections législatives de 2002 ", Revue française de science politique, vol.55, p.501-520.

Moreau Thérèse, Baider Fabienne, KhaznAdar Edwige éd., 2007, Nouvelles questions féministes, vol. 26, n³, "Parité linguistique ».

OLIVESI Aurélie, 2012, Implicitement sexiste? Genre, politique et discours journalistique, Toulouse, PUM.

PAoletti Marion, 2013, «Porte-parole dans la campagne présidentielle. Incarner son genre avec classe», Genre, sexualité \& société, Hors-série ${ }^{\circ}{ }^{2}$, "Présidentielle $2012 »$.

Restier-Melleray Christine, 1999, «La femme du présidentiable : une figurante engagée », Le métier politique en représentation, P. Mazet, Y. Poirmeur éd., Paris, L'Harmattan, p. 89-159.

Scott Joan, 1998, La citoyenne paradoxale. Les féministes françaises et les droits de l'homme, Paris, Albin Michel.

SINEAU Mariette, 1988, Des femmes en politique, Paris, Economica.

- 2008, La force du nombre. Femmes et démocratie présidentielle, Paris, L’Aube.

- 2011, Femmes et pouvoir sous la Ve République. De l'exclusion à l'entrée dans la course présidentielle, Paris, Presses de Sciences Po.

VASSY Serge, 2005, "Ethos de femmes ministres. Recherche d'indices quantifiables», Mots. Les langages du politique, $\mathrm{n}^{\circ} 70$, «Usages politiques du genre », p. 105-174.

Viennot Éliane, 2006 et 2008, La France, les femmes et le pouvoir, Paris, Perrin, 2 tomes. 
